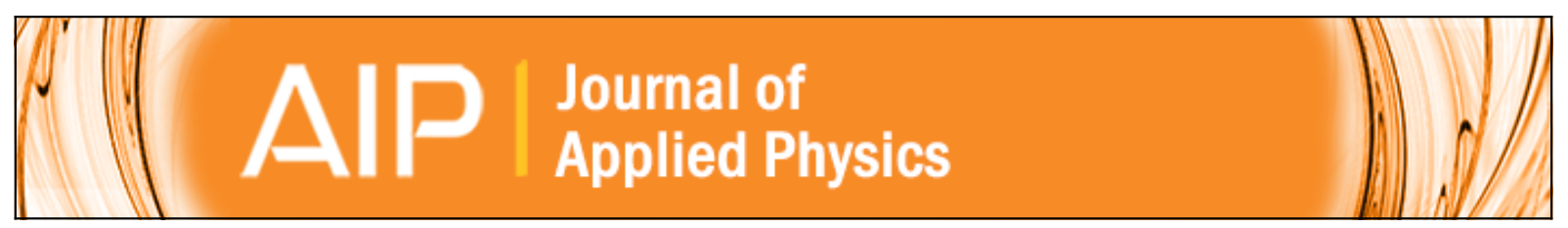

\title{
Single orientation graphene synthesized on iridium thin films grown by molecular beam epitaxy
}

A. Dangwal Pandey, K. Krausert, D. Franz, E. Grånäs, R. Shayduk, P. Müller, T. F. Keller, H. Noei, V. Vonk, and A. Stierle

Citation: Journal of Applied Physics 120, 075304 (2016); doi: 10.1063/1.4960804

View online: http://dx.doi.org/10.1063/1.4960804

View Table of Contents: http://scitation.aip.org/content/aip/journal/jap/120/7?ver=pdfcov

Published by the AIP Publishing

\section{Articles you may be interested in}

Structural properties of Bi2Te3 topological insulator thin films grown by molecular beam epitaxy on (111) BaF2 substrates

J. Appl. Phys. 119, 165303 (2016); 10.1063/1.4947266

Epitaxial (111) films of $\mathrm{Cu}, \mathrm{Ni}$, and CuxNiy on a-Al2O3 (0001) for graphene growth by chemical vapor deposition J. Appl. Phys. 112, 064317 (2012); 10.1063/1.4754013

Epitaxial graphene prepared by chemical vapor deposition on single crystal thin iridium films on sapphire Appl. Phys. Lett. 98, 181903 (2011); 10.1063/1.3585126

Single-crystalline aluminum grown on MgAl 204 spinel using molecular-beam epitaxy

J. Vac. Sci. Technol. B 29, 03C128 (2011); 10.1116/1.3570869

Defects in HgTe grown by molecular beam epitaxy on (211)B-oriented CdZnTe substrates

J. Vac. Sci. Technol. B 25, 1776 (2007); 10.1116/1.2787876

\section{HDDEN}

\section{Instruments for Advanced Science}

Contact Hiden Analytical for further details: w www.HidenAnalytical.com E info@hiden.co.uk CLICK TO VIEW our product catalogue

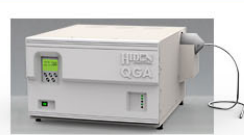

Gas Analysis

, dynamic measurement of reaction gas streams catalysis and thermal analysis ' molecular beam studies

'dissolved species probes

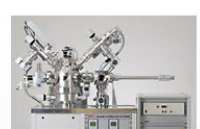

Surface Science

, UHVTPD

, SIMS
, end point detection in ion beam etch , elemental imaging - surface mapping

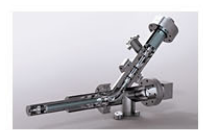

Plasma Diagnostics

, plasma source characterization etch and deposition process reaction , kinetic studies , analysis of neutral and radical species

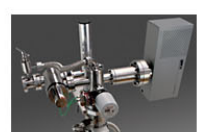

Vacuum Analysis ' partial pressure measurement and control of process gases , reactive sputter process control , vacuum coating process monitoring 


\title{
Single orientation graphene synthesized on iridium thin films grown by molecular beam epitaxy
}

\author{
A. Dangwal Pandey, ${ }^{1, a)}$ K. Krausert, ${ }^{1,2}$ D. Franz ${ }^{1,2}$ E. Grånäs, ${ }^{1}$ R. Shayduk, ${ }^{1}$ P. Müller, ${ }^{1,2}$ \\ T. F. Keller, ${ }^{1,2}$ H. Noei, ${ }^{1}$ V. Vonk, ${ }^{1}$ and A. Stierle ${ }^{1,2, b)}$ \\ ${ }^{1}$ Deutsches Elektronen-Synchrotron (DESY), D-22607 Hamburg, Germany \\ ${ }^{2}$ Fachbereich Physik, Universität Hamburg, D-22607 Hamburg, Germany
}

(Received 14 April 2016; accepted 11 July 2016; published online 16 August 2016)

\begin{abstract}
Heteroepitaxial iridium thin films were deposited on (0001) sapphire substrates by means of molecular beam epitaxy, and subsequently, one monolayer of graphene was synthesized by chemical vapor deposition. The influence of the growth parameters on the quality of the Ir films, as well as of graphene, was investigated systematically by means of low energy electron diffraction, $\mathrm{x}$-ray reflectivity, x-ray diffraction, Auger electron spectroscopy, scanning electron microscopy, and atomic force microscopy. Our study reveals (111) oriented iridium films with high crystalline quality and extremely low surface roughness, on which the formation of large-area epitaxial graphene is achieved. The presence of defects, like dislocations, twins, and $30^{\circ}$ rotated domains in the iridium films is also discussed. The coverage of graphene was found to be influenced by the presence of $30^{\circ}$ rotated domains in the $\mathrm{Ir}$ films. Low iridium deposition rates suppress these rotated domains and an almost complete coverage of graphene was obtained. This synthesis route yields inexpensive, air-stable, and large-area graphene with a well-defined orientation, making it accessible to a wider community of researchers for numerous experiments or applications, including those which use destructive analysis techniques or irreversible processes. Moreover, this approach can be used to tune the structural quality of graphene, allowing a systematic study of the influence of defects in various processes like intercalation below graphene. Published by AIP Publishing. [http://dx.doi.org/10.1063/1.4960804]
\end{abstract}

\section{INTRODUCTION}

The preparation of large-area graphene with high structural quality is in demand to exploit its two-dimensional nature with unique physical properties for diverse applications, including future electronic devices and sensors. ${ }^{1,2}$ Chemical vapor deposition (CVD) has become an established method to deposit epitaxial graphene on transition metal single crystals, ${ }^{3-5}$ and it was further demonstrated that epitaxial graphene with a preselected orientation can be synthesized using this route. ${ }^{6-10}$ CVD growth of graphene on Ir and Pt is self-limiting, which results exactly in one monolayer of graphene coverage of the surface. ${ }^{3,4,11}$ Graphene is found to interact weakly with the surfaces of $\operatorname{Ir}(111)$ and $\operatorname{Pt}(111)$ and is of high quality on these substrates. ${ }^{12-15}$ However, a weak interaction between graphene and support might favor the formation of different coexisting domains of graphene, ${ }^{16}$ for example, non-rotated graphene on Pt (111) with a periodicity of $2.2 \mathrm{~nm}, 1.5^{\circ}$ rotated graphene with a periodicity of $2 \mathrm{~nm}$, and a $90^{\circ}$ rotated graphene with a periodicity of $0.5 \mathrm{~nm} .{ }^{17} \mathrm{On} \operatorname{Ir}(111)$, the most abundant form of graphene is non-rotated (R0) with respect to its support, while $30^{\circ}$ rotated, $18.5^{\circ}$ rotated, and $14^{\circ}$ rotated forms of graphene are also reported. ${ }^{18}$ The non-rotated graphene can be grown as a single, millimeter sized, layer on $\operatorname{Ir}(111)$ single crystals, ${ }^{6}$ where the moire structure of R0 graphene exhibits a periodicity of $2.53 \mathrm{~nm}$ and $0.02 \mathrm{~nm}$ vertical corrugations. ${ }^{19,20}$ A lattice mismatch between the metal substrates

\footnotetext{
${ }^{\text {a)} E l e c t r o n i c ~ m a i l: ~ a r t i . p a n d e y @ d e s y . d e ~}$

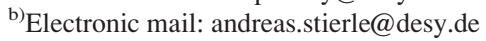

and graphene leads to a slight, but periodic, undulation of graphene on the top. These undulations in graphene are imaged by means of scanning tunneling microscopy (STM) as moiré structures. ${ }^{21,22}$ Graphene moiré on $\operatorname{Ir}(111)$ has been exploited as an active template to grow highly ordered lattice of variety of metal clusters such as Ir, Pt, W, Re, Au, and Iron. ${ }^{19,23}$ A regular array of monodispersed metal nanoclusters forms a model system to study various physical phenomenon or chemical processes like heterogeneous catalysis, electrical transport, magnetism, etc. However, here the use of expensive single crystals makes this synthesis route less convenient for a wide community of researchers to conduct various possible experiments on the nicely formed cluster lattice on graphene moiré. A possible approach to circumvent this problem is to synthesize epitaxial graphene on $\operatorname{Ir}(111)$ thin films grown on an inexpensive substrate. Only few studies were reported on synthesizing graphene on iridium films, where sapphire $(0001)^{24}$ or $\mathrm{Si}(111)$ with yttriastablized zirconia (YSZ) buffer layer ${ }^{25,26}$ was used as a support.

Sapphire crystals are widely used as substrates to grow metal films due to their high thermal and chemical stabilities, necessary for withstanding the harsh conditions of the CVD process required for the synthesis of high-quality graphene. ${ }^{27-30}$ Single crystal iridium(111) films can be deposited by pulsed laser deposition (PLD) ${ }^{24}$ and metal-organic $\mathrm{CVD}^{31}$ on (0001) sapphire single crystals. Deposition of epitaxial iridium films on other orientations of sapphire was also reported by using the method of metal-organic CVD and an epitaxial relationship between different orientations 
of the deposited film and the substrate was obtained from x-ray diffraction (XRD) measurements. ${ }^{31}$ Apart from these, the growth of (111) oriented Ir films on sapphire substrates is not explored systematically, and to the best of the authors' knowledge, no report on (111) oriented iridium films grown on c-plane sapphire substrates using molecular beam epitaxy (MBE) is available up to date.

Here, we report on the deposition of epitaxial $\operatorname{Ir}(111)$ thin films on (0001) oriented sapphire single crystals by means of MBE and its use as a support to synthesize graphene monolayer by CVD. Various techniques were employed to characterize the iridium films, grown with systematically varying deposition parameters, and the subsequently synthesized graphene. Low energy electron diffraction (LEED) and x-ray diffraction (XRD) confirm that the (111) oriented iridium films are highly crystalline, and X-ray reflectivity (XRR) measurements verify their extremely low surface roughness. The presence of defects like dislocations, and rotated and twin domains in the film were also studied and their influence on the graphene was observed. An almost full coverage of graphene on the iridium films was confirmed by Auger electron spectroscopy (AES), and its perfect alignment with respect to the underlying Ir lattice was verified by LEED. The surface morphology and topography mapped by means of scanning electron microscopy (SEM) and non-contact atomic force microscopy (NC-AFM) revealed a homogeneous coverage and an atomically smooth surface of graphene on top. This is further corroborated by the XRR measurements showing the signature of the graphene layer on top of the iridium film.

\section{EXPERIMENTAL}

Sapphire (0001) crystals with a surface area of 10 $\times 10 \mathrm{~mm}^{2}$ and low miscut $\left(<0.1^{\circ}\right)$ were used as substrates. The as-received crystals were broken into two parts (for growing two samples at the same time) and cleaned with acetone, ethanol, and isopropanol successively in an ultrasonic bath. Sapphire surfaces were prepared by heat treatment at $1000 \mathrm{~K}$ in UHV for $40 \mathrm{~min}$ and treatment with atomic oxygen from a thermal cracker, followed by a flash annealing above $1200 \mathrm{~K}$ in UHV. Iridium films of various thicknesses were deposited by molecular beam epitaxy in an UHV chamber (base pressure of $5 \times 10^{-11} \mathrm{mbar}$ ), using an e-beam evaporator. The growth rate was varied as summarized in Table I, between $0.06 \mathrm{~nm} / \mathrm{min}$ and $1.2 \mathrm{~nm} / \mathrm{min}$ for different iridium films. During deposition, all the samples were kept at $700 \mathrm{~K}$ unless mentioned otherwise. For the highest growth rate, the growth temperature was $800 \mathrm{~K}$. In another case (sample $13 \mathrm{~A}$ ), a differential growth temperature was used, i.e., $1000 \mathrm{~K}$ in the beginning for one third of the deposition time and $700 \mathrm{~K}$ for the rest of the deposition. A post-annealing treatment was employed for all samples by a successive heating at $1100 \mathrm{~K}$ for $30 \mathrm{~min}$. Graphene was synthesized by exposing the iridium film to ethylene with a partial pressure of $\sim 10^{-7}$ mbar until a complete coverage at room temperature and then at temperatures above $1050 \mathrm{~K}$. More details on graphene synthesis are given in Section III B.

LEED and AES measurements were performed in situ after each step of surface cleaning, iridium deposition, and graphene
TABLE I. Summary of X-ray reflectivity and longitudinal Bragg scans' results. The varied growth parameters for iridium films are growth temperature $T_{G}$ and growth rate $G$. The retrieved parameters are film thickness $t$, interface roughness $\sigma_{i}$, and film surface roughness $\sigma_{s}$ from X-ray reflectivity fits, out-of-plane coherence lengths $L_{\mathrm{c}}$ from out-of-plane Bragg scan, and inplane mosaicity $\Delta \theta$ from in-plane theta-scans.

\begin{tabular}{lccccccc}
\hline \hline $\begin{array}{l}\text { Nominal film } \\
\text { thickness }(\mathrm{nm})\end{array}$ & $\begin{array}{c}T_{G} \\
(\mathrm{~K})\end{array}$ & $\begin{array}{c}G \\
(\mathrm{~nm} / \mathrm{min})\end{array}$ & $\begin{array}{c}\sigma_{i} \\
(\mathrm{~nm})\end{array}$ & $\begin{array}{c}t \\
(\mathrm{~nm})\end{array}$ & $\begin{array}{c}\sigma_{\mathrm{s}} \\
(\mathrm{nm})\end{array}$ & $\begin{array}{c}L_{c} \\
(\mathrm{~nm})\end{array}$ & $\begin{array}{c}\Delta \theta \\
(\mathrm{deg})\end{array}$ \\
\hline 40 & 800 & 1.2 & 1.78 & 41.0 & 0.16 & 44.2 & 1.9 \\
30 & 700 & 0.6 & 1.52 & 26.9 & 0.095 & 26.8 & 1.07 \\
$15^{\mathrm{a}}$ & 700 & 0.22 & 0.15 & 14.8 & 0.05 & 14.8 & 0.78 \\
$13 \mathrm{~A}$ & 1000, & 0.19 & 0.17 & 12.5 & 0.050 & 12.7 & 0.51 \\
& 700 & & & & & & \\
$13 \mathrm{~B}$ & 700 & 0.15 & 0.19 & 13.1 & 0.055 & 13.3 & 0.62 \\
10 & 700 & 0.06 & 0.55 & 9.9 & 0.077 & 9.76 & $\ldots$ \\
\hline \hline
\end{tabular}

${ }^{\mathrm{a}}$ Ir film was graphene covered.

synthesis, to study the structure and composition of the surface. The deposited iridium films were contacted by a tantalum foil to minimize the charging of the insulating sapphire substrate during LEED measurements. For further characterization, the samples were stored under ambient conditions.

$\mathrm{X}$-ray reflectivity and diffraction experiments were conducted for a detailed structural characterization of the surface and bulk of the samples. X-ray reflectivity is used to determine the film thickness, surface roughness, interfacial roughness, and the electron density profile perpendicular to the film plane. Out-of-plane Bragg scans (or radial scans measured in high angle regime) at a reciprocal-lattice point along the growth direction of the film yields information on the crystalline perfection and mosaicity. Specular rods were simulated by using the software ROD. ${ }^{32}$ In-plane Bragg scans, performed at a grazing angle of incidence equal to the critical angle for the total external reflection for iridium to achieve a maximum signal to noise ratio, are used to determine the epitaxial relationship between the deposited iridium film and the substrate, average in-plane crystallite size, and mosaicity. A four-circle reflectometer with a $\mathrm{Mo}_{\alpha}$ source and a six-circle diffractometer with $\mathrm{Cu} \mathrm{K}_{\alpha}$ source were used for $\mathrm{x}$-ray reflectivity and diffraction measurements.

To probe the reciprocal space of the $\operatorname{Ir}(111)$ film, an orientation matrix was set in terms of hexagonal surface coordinates, with lattice parameters: $a=b=\frac{a_{0}}{\sqrt{2}}, c=a_{0} \cdot \sqrt{3}$, and $\alpha=\beta=90^{\circ}, \gamma=120^{\circ}$ with Ir bulk lattice parameter $a_{0}=3.8392 \AA$. In this article, we used the notation $(h k l)$ for bulk coordinates and $(H K L)_{s}$ for surface co-ordinates of the (111) oriented face centered cubic (fcc) iridium films. The transformation between bulk and surface co-ordinates is as follows:

$$
\left(\begin{array}{l}
h \\
k \\
l
\end{array}\right)=\frac{1}{3}\left(\begin{array}{ccc}
2 & -2 & 1 \\
2 & 4 & 1 \\
-4 & -2 & 1
\end{array}\right)\left(\begin{array}{l}
H \\
K \\
L
\end{array}\right)_{s} .
$$

The surface topography of a fresh graphene covered iridium film was measured at room temperature by NC-AFM 
with a base pressure of $<5 \times 10^{-11}$ mbar. Some samples were also examined by NC-AFM under ambient conditions. The surface morphology of bare and graphene covered iridium films was additionally characterized using SEM.

\section{RESULTS AND DISCUSSION}

\section{A. Growth of iridium films on (0001) sapphire}

The cleaned and annealed sapphire crystal surface shows a bright $(1 \times 1)$ LEED pattern with clear threefold symmetry. Well-defined hexagonal spots in the LEED pattern were obtained after the iridium deposition, as given in Fig. 1(a), in line with a (111) oriented surface. In a particular case, when the iridium film was deposited at a high growth rate of $1.2 \mathrm{~nm} / \mathrm{min}$, additional LEED spots rotated by $30^{\circ}$ with respect to the normal LEED pattern were observed (Fig. 1(b)), which verify the existence of $30^{\circ}$ rotated domains in the film.

AES data were collected from an annealed sapphire crystal surface before and after the deposition of iridium films, as given in Fig. 1(c). For a comparison, an Auger spectrum from an $\operatorname{Ir}(111)$ single crystal, measured in the same set-up, is also plotted in Fig. 1(c). The bare sapphire shows no contaminants, and after the deposition of iridium, the characteristic peaks for iridium, comparable to that from an $\operatorname{Ir}(111)$ single crystal, are observed, while the AES oxygen KLL signal from the sapphire substrate is completely absent. The absence of the O KLL signal demonstrates that the iridium film deposited on sapphire is completely closed. A shift of about $4 \mathrm{eV}$ in the spectrum of the Ir-film arises because of charging of the film deposited on an insulating substrate.

NC-AFM investigations were performed ex situ to image the surface topography of the iridium films. Fig. 1(d) shows the scanned area of $2.2 \times 2.2 \mu \mathrm{m}^{2}$ over a $10 \mathrm{~nm}$ thick iridium film. The film surface is relatively smooth as depicted from the roughness profile, and the root mean square roughness value of $0.15 \mathrm{~nm}$ was obtained for the film surface.

\section{X-ray reflectivity studies}

The x-ray reflectivity curves show the intensity of specularly reflected x-rays at small angles, which depend on the average electron density profile of the material perpendicular to its surface. Figure 2 shows the reflectivity data for iridium films with varying thickness from $10 \mathrm{~nm}$ to $40 \mathrm{~nm}$ together with their respective fits. For a better comparison, the intensity is plotted as a function of $\mathrm{Q}$. Iridium films of $13 \mathrm{~nm}$ and $10 \mathrm{~nm}$ show distinct oscillations in the reflectivity curves, while thickness oscillations are strongly damped for $30 \mathrm{~nm}$ and $40 \mathrm{~nm}$ iridium films. To fit the reflectivity data, we used Parratt formalism for x-ray reflectivity, ${ }^{33}$ with a modification to include the contribution of roughness at the interfaces. ${ }^{34}$ In the case of damped reflectivity oscillations, the film thickness was determined by using the Scherrer equation ${ }^{35}$ for the $\operatorname{Ir}(111)$ reflection, and this value was used for the fit. For the electron density of the iridium film, we have assumed the bulk value. The parameters deduced from the fit are summarized in Table I.

On comparing the surface roughness of all iridium films, we find that the highest value of $0.16 \mathrm{~nm}$ is observed for the
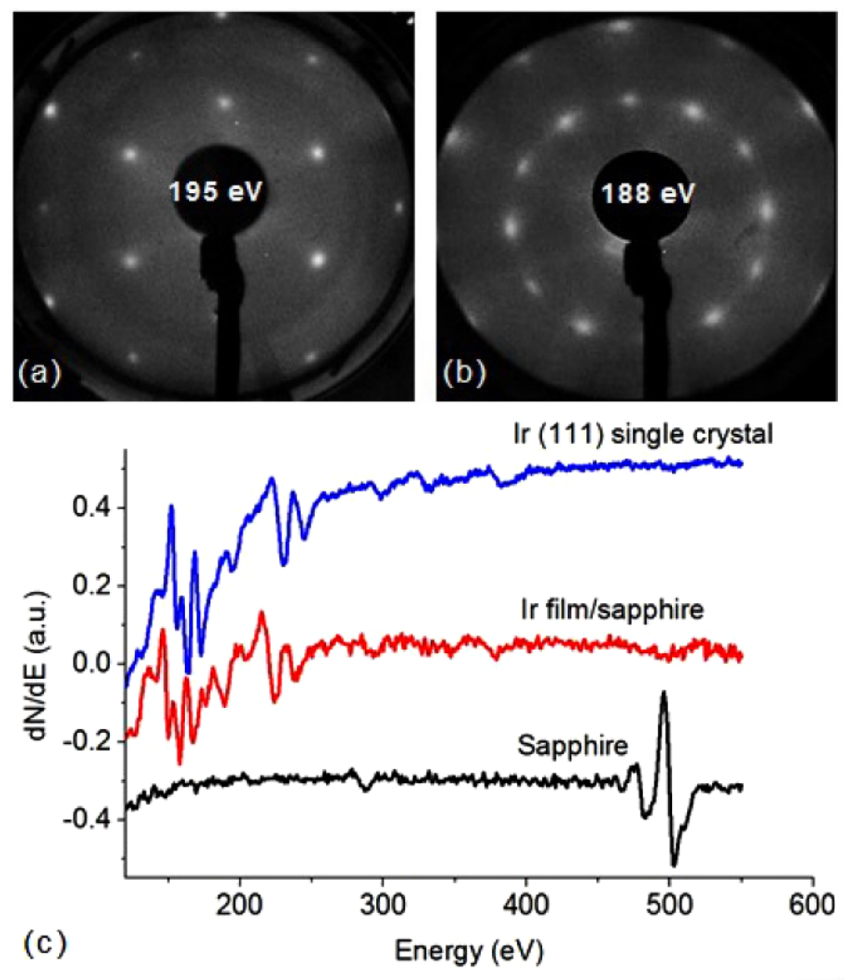

$\mathrm{nm}$

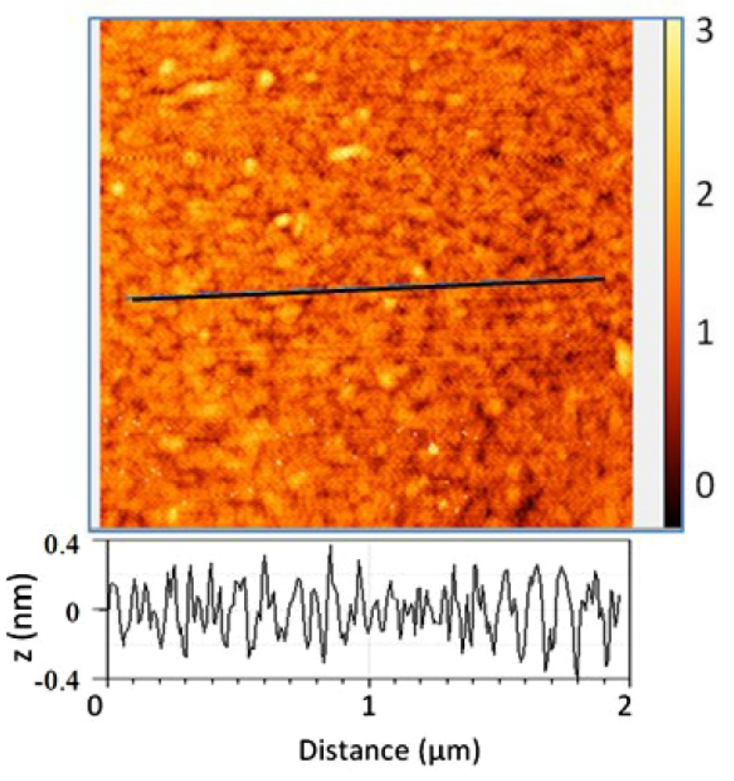

(d)

Distance $(\mu \mathrm{m})$

FIG. 1. (a) LEED pattern of an iridium film deposited with a growth rate of $0.2 \mathrm{~nm} / \mathrm{min}$ and (b) $1.2 \mathrm{~nm} / \mathrm{min}$. (c) A typical Auger electron spectrum for cleaned sapphire crystal (in black), deposited iridium films (in red), and compared to an iridium (111) single crystal (in blue). For clarity, the curves are shifted in vertical axis. (d) Surface topography measured by means of inair NC-AFM over a $2.2 \times 2.2 \mu \mathrm{m}^{2}$ area on a $10 \mathrm{~nm}$ iridium film, the roughness profile along the line is shown at the bottom.

film grown with high deposition rate $(1.2 \mathrm{~nm} / \mathrm{min})$, while extremely smooth surfaces are achieved (surface roughness $<0.1 \mathrm{~nm}$ ) for the films deposited with a rate of $0.6 \mathrm{~nm} / \mathrm{min}$ or lower. The in-air NC-AFM results on a $10 \mathrm{~nm}$ iridium film grown with a rate of $0.06 \mathrm{~nm} / \mathrm{min}$, as shown in Fig. 1(d), provide similar roughness values. Since the microscopy techniques probe the sample locally, we believe that the surfaces of iridium films deposited on sapphire are extremely smooth 


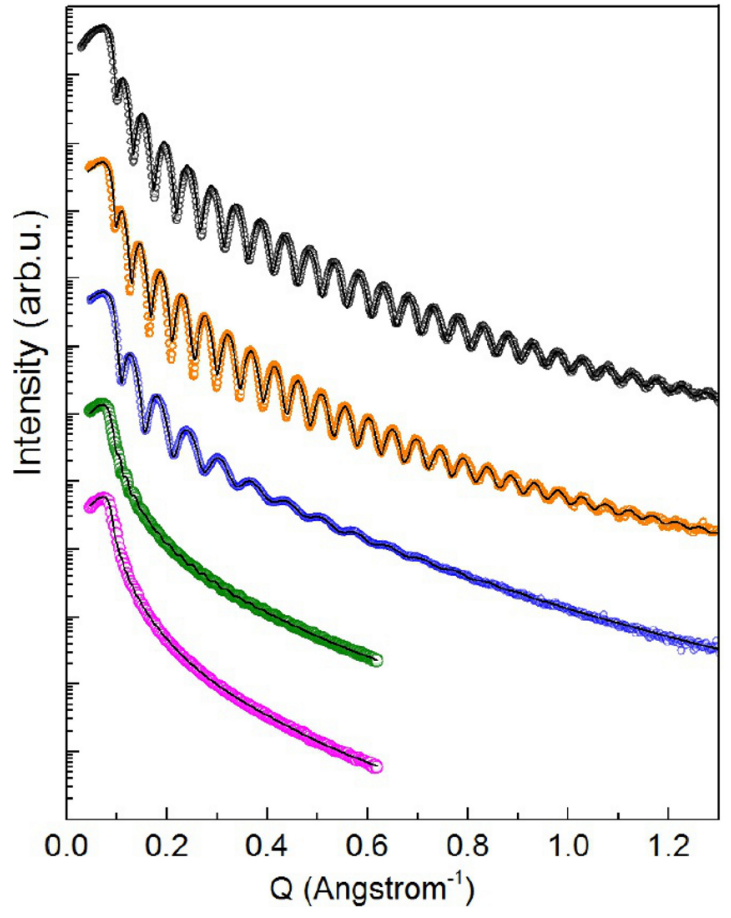

FIG. 2. X-ray reflectivity curves for iridium films of different thicknesses. From the bottom and up: $40 \mathrm{~nm}$ (magenta), $30 \mathrm{~nm}$ (green), $10 \mathrm{~nm}$ (blue), and $13 \mathrm{~nm}$ (A-orange and B-black) Ir films. Circles are data points and lines are fit of the data using the Parratt formalism. Both $13 \mathrm{~nm}$ films were measured using $\mathrm{Cu} \mathrm{K} \alpha$ radiation, while Mo $\mathrm{K} \alpha$ was used for rest of the samples.

over the entire surface as depicted by XRR measurements, which is advantageous for successive high-quality graphene growth.

Two samples of $13 \mathrm{~nm}$ iridium film thickness were grown with a similar deposition rate but at different growth temperatures: one at $700 \mathrm{~K}$ and the other at $1000 \mathrm{~K}$ for one third of deposition time and at $700 \mathrm{~K}$ afterwards. In the latter case, a higher growth temperature in the beginning of the growth process was intended to study its influence on the crystallinity of the film. In both cases, clear oscillations in a wide angular range are observed, and extremely low values for surface roughness $(<0.06 \mathrm{~nm})$ are obtained. Moreover, a small interface roughness $(<0.2 \mathrm{~nm})$ between iridium films and sapphire matches closely to the atomic step height in (0001) sapphire crystal surface.

The origin of damped reflectivity oscillations for $30 \mathrm{~nm}$ and $40 \mathrm{~nm}$ iridium films is found to be a gradient in the film thickness due to a small distance between the sample and the Ir-evaporator during the film growth. This was confirmed by performing radial Bragg scans with smaller x-ray beam footprint at different points on the film surface and the variation in the film thickness up to $\sim 1 \mathrm{~nm} / \mathrm{mm}$ was obtained. The very high values of the interface roughness $(>1.5 \mathrm{~nm})$ for these films are artefacts that are caused by the thickness gradients leading to a smearing out of the thickness oscillations.

\section{Out-of-plane Bragg scan results}

Crystalline thin films, when consisting of $N$ coherently scattering lattice planes, are characterized by Bragg reflections with satellite fringes of Laue oscillations given by $I=\sin ^{2}\left(Q L_{c} / 2\right) / \sin ^{2}(Q d / 2)$, where $d$ is the interplanar spacing corresponding to the angular position of Bragg reflection, $L_{c}=N \cdot d$ is the coherent thickness, and $Q=\left(\frac{4 \pi}{\lambda}\right) \sin \alpha$ is the magnitude of the scattering vector.

Fig. 3 shows a radial Bragg scan through the $\operatorname{Ir}(111)$ reflection for a $13 \mathrm{~nm}$ iridium film. The observed out-ofplane epitaxial relationship is $\operatorname{Ir}(111) \| \mathrm{Al}_{2} \mathrm{O}_{3}(0001)$. The experimental $d_{111}$ value is $2.2187( \pm 0.0015) \AA$, which is close to that for bulk iridium $(2.2167 \AA)$. Laue oscillations of the $\operatorname{Ir}(111)$ Bragg reflection are very prominent in these measurements. $L_{c}$ is calculated to be $13.3 \mathrm{~nm}$ from the period of Laue oscillations, which is approximately identical to the thickness of the film retrieved from the reflectivity curve. The coherence thicknesses obtained for different films are summarized in Table I, matching closely to the film thicknesses retrieved from the reflectivity measurements. This confirms that these iridium films are completely made up of coherently scattering (111) planes in the vertical direction.

A rocking scan obtained with a fixed detector at the position for the $\operatorname{Ir}(111)$ reflection is given in the inset of Fig. 3, which provides information on the mosaicity normal to film plane. This rocking curve shows two components: a resolution limited sharp Bragg component with FWHM of $0.12^{\circ}$ superimposed on a broader diffuse component with FWHM of $\sim 0.54^{\circ}$. Lattice distortions are found to be responsible for giving rise to such a broad diffuse component in the rocking scans of epitaxial films. ${ }^{36}$ The lateral coherence length of $23 \mathrm{~nm}$ is derived from the FWHM of the diffuse component, which represents the average distance between dislocations in the film. The presence of structural defects (such as dislocations, local deformation of lattice planes, and twinned domains), based on x-ray and microscopy measurements, was reported for epitaxially grown (111) oriented fcc metal films on sapphire substrates. ${ }^{37,38}$ The existence of twinned domains in the here discussed iridium films will be discussed in the following subsection.

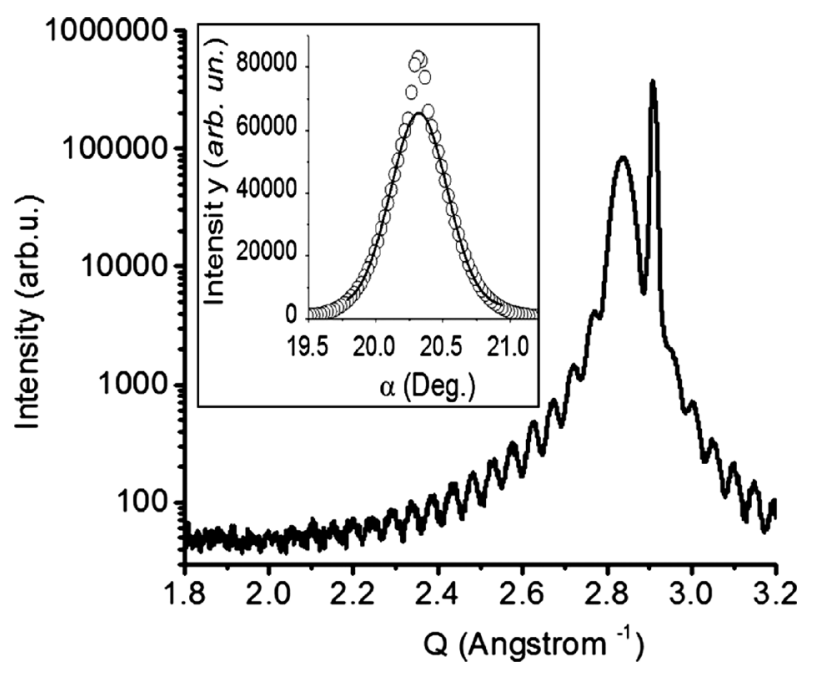

FIG. 3. Radial Bragg scan of $\operatorname{Ir}(111)$ reflection for a $13 \mathrm{~nm}$ iridium film. Inset: rocking curve at $\operatorname{Ir}(111)$ Bragg peak. The data points are indicated with circles and the fit to the broad component is indicated with line. 


\section{Grazing incidence $x$-ray diffraction results}

To characterize the in-plane structure of the Ir films in detail, we performed in-plane Bragg scans at a grazing angle of $0.597^{\circ}$, which is the critical angle of incidence for total external reflection by iridium for $\mathrm{Cu} K_{\alpha}$ radiation. Figure 4 shows a plot of theta-scans for iridium films, in which the sample was rotated around the film surface normal and the detector was kept fixed at the $\operatorname{Ir}(2 \overline{2} 0)$ Bragg peak position. We observe that main Bragg reflections appear around $-85^{\circ}$, $-25^{\circ}, 35^{\circ}, 95^{\circ}$, and $155^{\circ}$, in line with the six-fold symmetry of $(2 \overline{2} 0)$ and equivalent reflections. The variation in the intensities of reflections is caused by the rectangular geometry of the sample. A $40 \mathrm{~nm}$ iridium film grown at the highest deposition rate of $1.2 \mathrm{~nm} / \mathrm{min}$ showed intense reflections at $30^{\circ}$ intervals in addition to the $60^{\circ}$ reflections. This is in line with the LEED observations on this sample (Fig. 1(b)), indicating that the $30^{\circ}$ rotated domains exist over the whole film thickness. In the case of a $15 \mathrm{~nm}$ film deposited with a rate of $\sim 0.2 \mathrm{~nm} / \mathrm{min}$, no additional $30^{\circ}$ reflections were observed, suggesting the suppression of $30^{\circ}$ rotated domains in the iridium films by lowering the growth rate. The in-plane mosaicity for all iridium films, as derived from the FWHM of the in-plane reflections, lies within the range of $0.6-1^{\circ}$. A higher value of $1.9^{\circ}$ is obtained for the $40 \mathrm{~nm}$ Ir film with $30^{\circ}$ rotated domains (see Table I).

The in-plane epitaxial relationship for the here discussed Ir film on (0001) sapphire is $\operatorname{Ir}[1 \overline{2} 1] \| \mathrm{Al}_{2} \mathrm{O}_{3}[11 \overline{2} 0]$. The inplane radial scan for a $13 \mathrm{~nm}$ iridium film at the $(2 \overline{2} 0)$ bulk reflection $\left((110)_{S}\right)$ provides a value of $d_{2 \overline{2} 0}=1.354( \pm 0.001)$ $\AA$, close to the bulk value for iridium $(1.3574 \AA$ ), and $\sim 1.5 \%$ smaller than $d_{30 \overline{3} 0}$ of the sapphire substrate.

It has been reported that homo- or heteroepitaxial $\operatorname{Ir}(111)$ films grown by physical vapor deposition (PVD) suffer from twinning. ${ }^{31,39}$ The presence of twin-domains can be investigated by some particular line scans in reciprocal space with momentum transfer perpendicular to the surface. Fig. 5(b) shows a sketch of the Bragg reflections in surface coordinates for a (111) oriented fcc surface: black dots indicate reflections from $\mathrm{ABC}$ stacked regular domains and orange dots indicate reflections from CBA stacked twinned

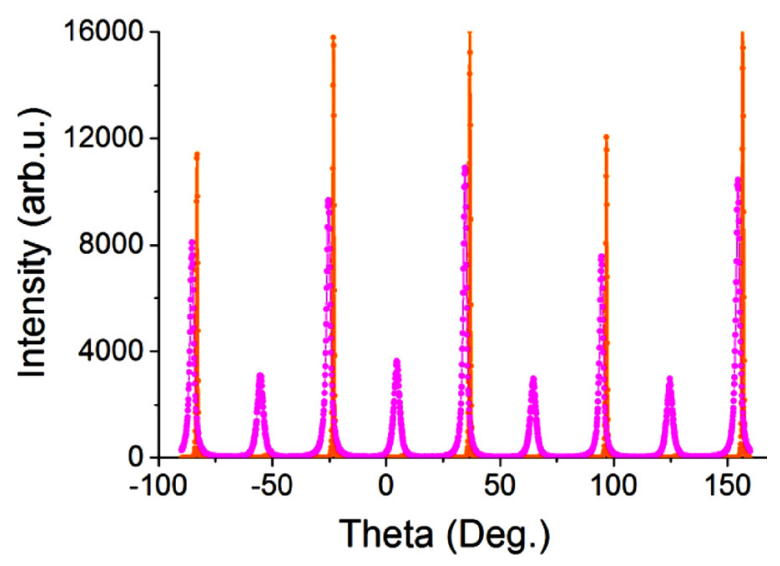

FIG. 4. Theta-scan of Ir (220) in-plane reflections indicating a six-fold symmetry for iridium films of $13 \mathrm{~nm}$ thickness (orange) and $40 \mathrm{~nm}$ thickness (magenta). Additional $30^{\circ}$ reflections are observed for $40 \mathrm{~nm}$ iridium film.
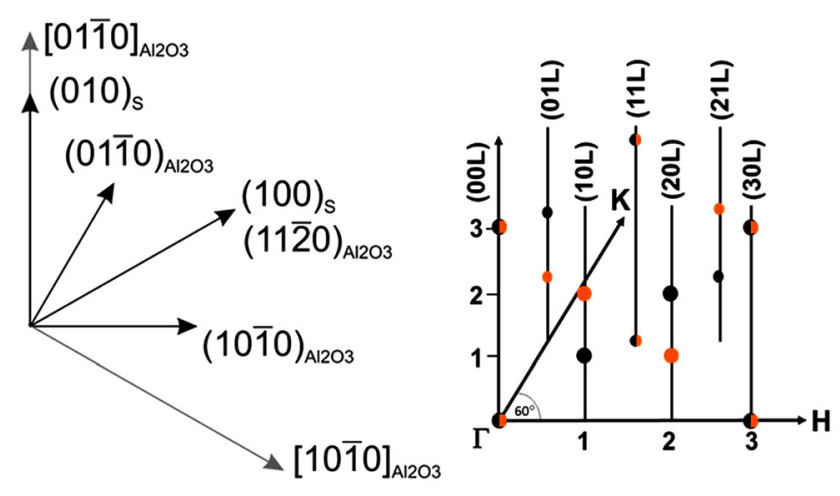

(a)

(b)
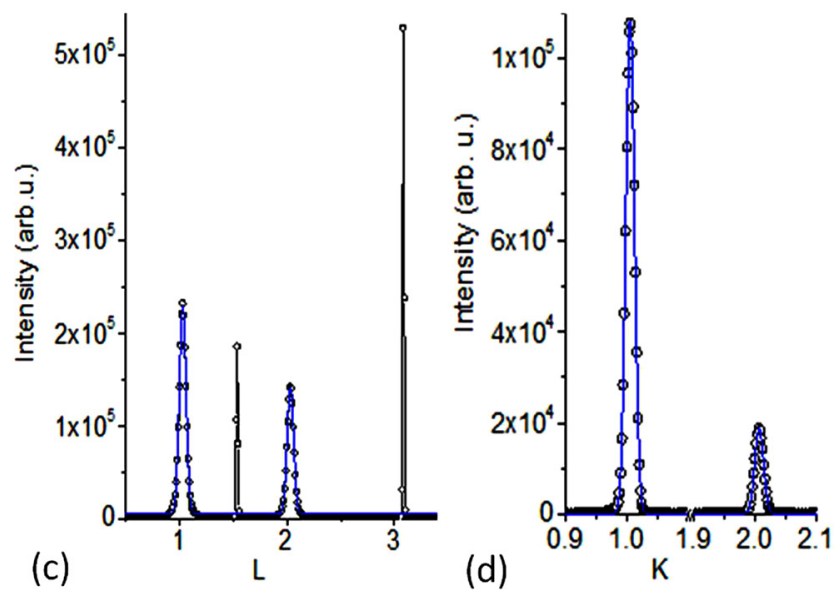

FIG. 5. (a) Sketch of the $\mathrm{Al}_{2} \mathrm{O}_{3}(0001) / \mathrm{Ir}(111)$ in-plane reciprocal lattice together with the $\mathrm{Al}_{2} \mathrm{O}_{3}$ real space directions (in [] brackets). (b) Sketch of Bragg reflections: black dots for an ideal (111) oriented fcc film, and blackorange dots for a film with 60 degree twinned domains. (c) L-scan along $\left(\begin{array}{lll}1 & 0 & L\end{array}\right)_{s}$ with $\mathrm{Ir}$ reflections at $\mathrm{L}=1$ and 2 . The sharp reflections at $\mathrm{L}=1.5$ and 3 arise from the sapphire substrate. (d) K-scan along $(0 \mathrm{~K} 1)_{\mathrm{s}}$. Data (black circles) in (c) and (d) are fitted (blue curve) to calculate the FWHM of the iridium Bragg peaks.

domains. From this, one can easily recognize that Bragg reflections from normal and twinned domains overlap for $L=3 n$ but are separated for $L \neq 3 n$.

A scan along the $(10 \mathrm{~L})_{\mathrm{s}}$ rod, performed on a $13 \mathrm{~nm}$ iridium film grown at $700 \mathrm{~K}$, is shown in Fig. 5(c). The presence of $(101)_{\mathrm{s}}$ and $(102)_{\mathrm{s}}$ Bragg reflections confirms the existence of twinned domains in the film. The same observation was made for all iridium films investigated in this study. No influence of deposition temperature and growth rates was found on the $60^{\circ}$ twinning in the iridium films.

On recently reported epitaxial iridium films on (0001) sapphire, grown by pulsed laser deposition at a substrate temperature of $700 \mathrm{~K}$, the annealing treatment of the films to $1100 \mathrm{~K}$ successfully removed twin formation. ${ }^{24}$ From the pole figure in Figure 1(a) of Ref. 24, it becomes evident that after the procedure discussed in Ref. 24, reciprocal planes with threefold symmetry around the surface normal are parallel for $\mathrm{Al}_{2} \mathrm{O}_{3}(0001)$ and $\operatorname{Ir}(111)$. This corresponds to an epitaxial relationship of $\operatorname{Ir}[1 \overline{2} 1] \| \mathrm{Al}_{2} \mathrm{O}_{3}[10 \overline{1} 0]$, which is exactly $30^{\circ}$ rotated compared to the epitaxial relationship found in our study after MBE growth.

We may speculate that the difference in in-plane orientation is responsible for the removal of the twinning in PLD 
grown heteroepitaxial iridium films because of a change in interfacial energy. Other factors such as differences in the growth mode between PLD and MBE, a different miscut, or different pre-treatment of the substrates might be responsible for the observed variation in in-plane orientation.

From the FWHM of the (101)s reflection along L, an outof-plane crystallite size of $9.2 \mathrm{~nm}$ is retrieved, which is significantly smaller than the total film thickness. Dislocations or stacking faults might be responsible for a reduced crystallite size along the film normal compared to the total film thickness. The scan given in Fig. 5(d) provides information on the in-plane domain size. The FWHM of the $(011)_{\mathrm{s}}$ reflection along $\mathrm{K}$ is 0.0165 and corresponds to an in-plane crystallite size of $16.45 \mathrm{~nm}$. This is close to the average distance between the dislocations/defects obtained from the out-of-plane rocking scan of the $\operatorname{Ir}(111)$ reflection. Moreover, approximately the same values of FWHMs of $\mathrm{K}$ scans, $\Delta \mathrm{K}=0.0165$ at $\mathrm{K}=1$ and $\Delta \mathrm{K}=0.0166$ at $\mathrm{K}=2$, suggest that the film is nearly free from in-plane strain gradients.

\section{B. Graphene synthesis on Ir(111) film}

After the deposition of highly crystalline $\operatorname{Ir}(111)$ films with atomically flat surfaces, we synthesized graphene by exposing the films at room temperature to ethylene until saturation $\left(10^{-7} \mathrm{mbar}\right)$, followed by annealing at very high temperatures $(>1200 \mathrm{~K})$ for aligning graphene domains parallel to the surface lattice. Further exposure of ethylene was performed in the range 1050-1150 K CVD temperature for $40 \mathrm{~min}$ to achieve maximum graphene coverage on the sample surface. For graphene growth on iridium single crystals, the reported annealing and CVD temperatures are $\sim 1500 \mathrm{~K}$ and $\sim 1000 \mathrm{~K}$, respectively. ${ }^{40}$ Due to a mismatch in the thermal expansion coefficients of sapphire and iridium, we expect crack formation in the iridium film by the rapid temperature changes. For this reason, we have modified the temperatures for the graphene growth. In the case of iridium films with $15 \mathrm{~nm}, 12 \mathrm{~nm}$, and $40 \mathrm{~nm}$ thickness, we used an annealing temperature of $1220 \mathrm{~K}$ and a CVD temperature of $1120 \mathrm{~K}$, while $30 \mathrm{~nm}$ and $10 \mathrm{~nm}$ thick iridium films were treated at an annealing temperature of $1250 \mathrm{~K}$ and a CVD temperature of $1050 \mathrm{~K}$ for graphene synthesis.

LEED patterns after graphene growth on a $15 \mathrm{~nm}$ film are given in Fig. 6(a), which shows clearly the formation of "R0' graphene, non-rotated with respect to the $\operatorname{Ir}(111)$ film. No other orientations of graphene are observed, which were reported for graphene growth at lower temperatures on Ir(111) single crystals. ${ }^{16,18}$ Distinct hexagonal spots are observed as a signature of multiple diffraction events, which are typical for electron diffraction. For a comparison with graphene grown on a $\operatorname{Ir}(111)$ single crystal, a corresponding LEED pattern is also given in Fig. 6(b). We observe that each hexagonal spot is slightly elongated in the tangential direction for graphene on iridium films, while the spots are circular in the case of iridium single crystal. This suggests the formation of slightly less-ordered graphene domains on iridium films, analogous to graphene grown on $\operatorname{Ir}(111)$ crystals at temperatures below $1300 \mathrm{~K} .^{41}$ To check the graphene quality over the whole sample, the electron beam was
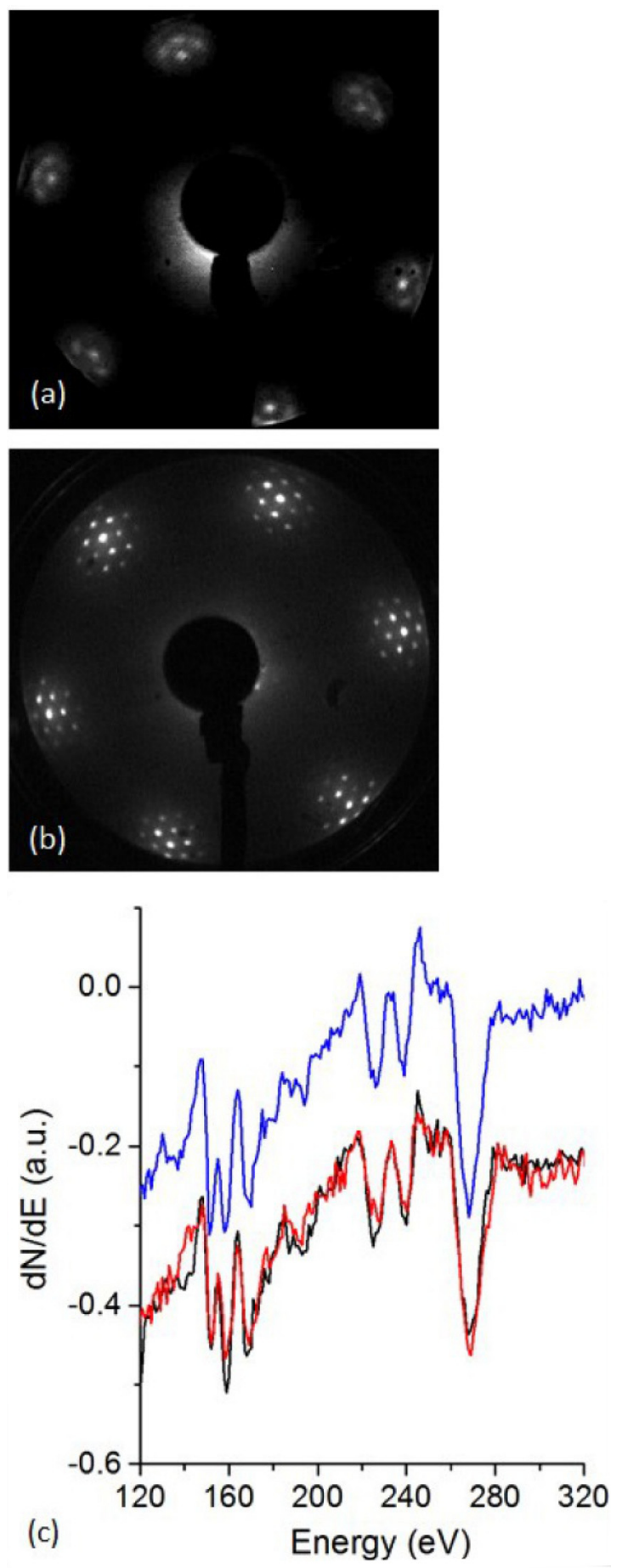

FIG. 6. LEED patterns at $75 \mathrm{eV}$ after graphene was synthesized on $15 \mathrm{~nm}$ iridium film (a) and bulk iridium single crystal (b). (c) Auger spectra measured on graphene deposited on a $15 \mathrm{~nm}$ iridium film (in red), $40 \mathrm{~nm}$ iridium film (in black), and $\operatorname{Ir}(111)$ single crystal (in blue).

scanned in steps over the whole sample and similar LEED patterns were recorded at all positions. This suggests the presence of a homogeneous, non-rotated, graphene layer formed on top of the iridium film.

The chemical composition of the surface was checked before and after graphene synthesis, by means of AES measurements (Fig. 6(c)), and the results were compared with graphene synthesized on an $\operatorname{Ir}(111)$ single crystal. The appearance of the KLL transition at $270 \mathrm{eV}$ corresponds to 
carbon and is in line with graphene formation. It was demonstrated that almost $100 \%$ full coverage of one monolayer graphene is achieved on $\operatorname{Ir}(111)$ single crystals with the described procedure. ${ }^{42}$ Identical heights of the C-peak for graphene on a $15 \mathrm{~nm}$ Ir film and graphene on an Ir single crystal suggests nearly full coverage of the iridium film by graphene, mainly limited by possible defects present on the film surface. On the other hand, a slightly smaller C-peak and higher Ir peaks are observed for the $40 \mathrm{~nm}$ iridium film, containing rotated domains, which hints for an incomplete graphene coverage on its surface; possibly, the C-coverage is limited by the larger amount of grain boundaries.

The surface topography of a freshly prepared graphene layer on top of a $13 \mathrm{~nm}$ iridium film is shown in Fig. 7, which was measured by NC-AFM in UHV. The straight line marked by an arrow most probably represents an atomic step originating from the sapphire miscut. The image reveals a rather high density of steps on the graphene covered Ir surface, with the steps not showing any clear preferential direction. Some islands exhibit edges which are rotated $120^{\circ}$ with respect to each other, while others appear more curved. The angular edges are typical for $\operatorname{Ir}(111)$ islands, while the curved features most probably correspond to the iridium grain boundaries. These observations of angular and curved edges are similar to what has been reported for insufficiently annealed pulsed laser deposited iridium films containing the twinned domains ${ }^{24}$ and also for graphene grown on $\mathrm{Cu}(111)$ on sapphire prior to annealing. ${ }^{27}$ The domain size of the $\mathrm{Ir}$ islands as observed in the NC-AFM images lie between $10 \mathrm{~nm}$ and $100 \mathrm{~nm}$, while in-plane domain size retrieved from XRD investigations is $\sim 14 \mathrm{~nm}$. This difference can be understood from the fact that XRD experiments provide representative information about the whole sample, while AFM measurements give the local information about the probed area, which is $500 \mathrm{~nm} \times 500 \mathrm{~nm}$ in this case.

Graphene seems to follow the iridium film topography consistently with an atomically smooth surface over the grains, depicting its perfect orientation with respect to the substrate. There are also some regions, as indicated by dotted lines in the AFM micrograph, where the surface appears significantly rougher, as seen in the corresponding line scan.
Higher roughness might come from not-perfectly grown graphene domains due to the defects on the iridium film surface, or adsorbates on those regions. More investigation is needed to understand this in detail. A line scan along a segment crossing two grains evidences atomically smooth area on both sides of the step. The height of the steps in both of the line scans is $\sim 0.22 \mathrm{~nm}$ and corresponds to an iridium-oniridium atomic step height.

Large-area surface morphologies for different samples were investigated via SEM before and after graphene synthesis. A homogeneous surface was observed for all of the as-deposited iridium films. After the graphene synthesis, a non-homogeneous surface was obtained for the $40 \mathrm{~nm}$ iridium film with rotated domains (Fig. 8(a)), indicating a partial graphene coverage, in line with the AES results from this sample. In contrast to this, the film surface was homogeneous for rest of the samples after graphene growth, as shown in Fig. 8(b) for the $10 \mathrm{~nm}$ iridium film. In a large area SEM micrograph (Fig. 8(c)) of the $10 \mathrm{~nm}$ iridium film, a high number of defects are observed on the surface. In-air AFM measurements revealed the presence of holes of approximately $1 \mu \mathrm{m}$ width in these areas of the film, as shown in Fig. 8(d). We believe this to be a signature of dewetting of the iridium film arising from the rapid temperature changes during the CVD growth of graphene. Large dewetting features of $\sim 50 \mu \mathrm{m}$ size have been observed by SEM on high-temperature annealed films of $\mathrm{Cu}(111)$ grown on sapphire ${ }^{27}$ and $\operatorname{Ir}(111)$ grown on YSZ/Si(111). ${ }^{25}$ The dewetting of the films could be avoided by an additional acid cleaning of sapphire in the former case, and use of thicker YSZ buffer layer on $\mathrm{Si}(111)$ in the latter case. For here discussed iridium films, growing thicker films might be helpful to overcome dewetting problem and investigations are going on in this direction.

$\mathrm{X}$-ray scattering measurements on the graphene covered sample provide details on the crystalline quality of the film after going through harsh CVD process for graphene synthesis. The reflectivity curve measured on a graphene covered $15 \mathrm{~nm}$ thick iridium film is shown in Fig. 9(a). The data are fitted with a model of a single layer graphene on an iridium film on a sapphire substrate. An excellent fit to the data was

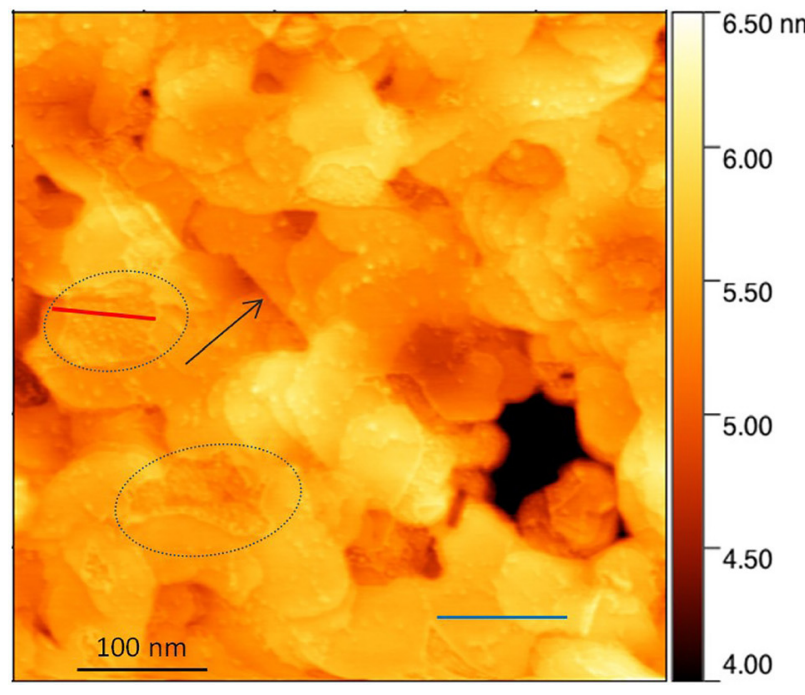

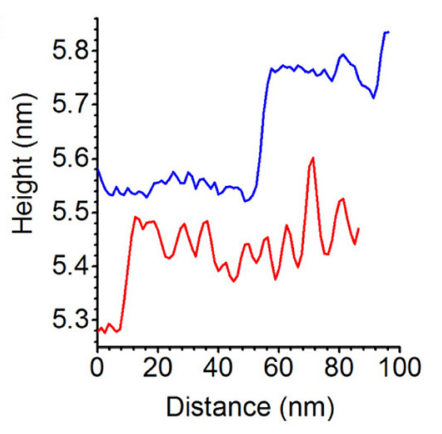

FIG. 7. AFM topograph of graphene surface on a $13 \mathrm{~nm}$ iridium film on sapphire. Image size: $500 \mathrm{~nm} \times 500 \mathrm{~nm}$. Height profiles along the blue and red lines are given on right. 

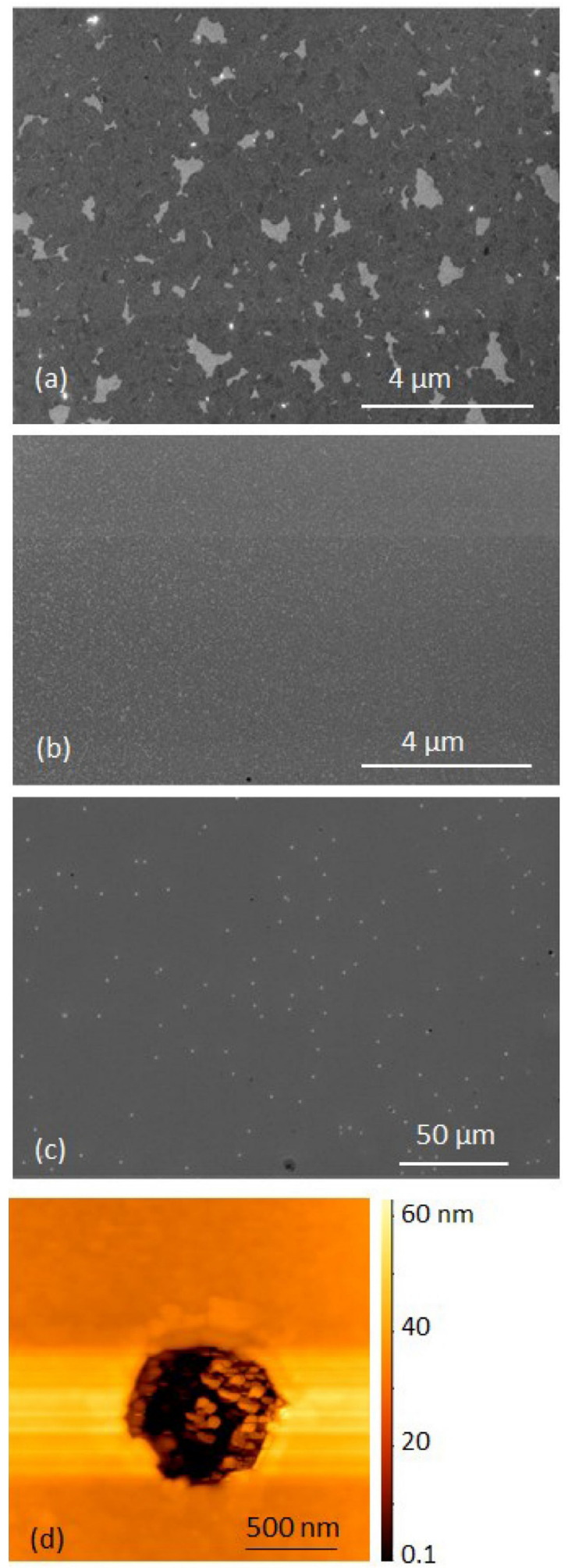

FIG. 8. SEM observations on graphene covered iridium films (a) of $40 \mathrm{~nm}$ and $(b, c)$ of $10 \mathrm{~nm}$. (d) In-air NC-AFM surface topography over one of the defects on $10 \mathrm{~nm}$ iridium film; scan size: $2 \mu \mathrm{m} \times 2 \mu \mathrm{m}$.

achieved for an iridium film thickness of $14.78 \mathrm{~nm}$ with surface roughness of $0.05 \mathrm{~nm}$ and interface roughness of $0.15 \mathrm{~nm}$, graphene thickness of $0.4 \mathrm{~nm}$ with surface roughness $0.17 \mathrm{~nm}$. The profile of electron density perpendicular to the surface of graphene on iridium film on sapphire is derived from the reflectivity fit and is given in the inset of
Fig. 9(a). In this plot, the electron density is given in terms of $2 \delta$, where $\delta$ is the decrement in the refractive index for $\mathrm{x}$-rays due to dispersion. To check the sensitivity of $\mathrm{x}$-ray reflectivity measurements to a single graphene monolayer, a simulated curve is also plotted in Figure 9(a) for the same iridium film on sapphire without considering the graphene layer on top. The difference is observable at higher incidence angles, where the reflectivity curve seems to be modulated by the presence of graphene layer. Interestingly, the distance between graphene to iridium top layer retrieved from the fit is $\sim 20 \%$ higher than that for graphene on $\operatorname{Ir}(111)$ single crystals. The presence of defects such as grain boundaries on the film surface might be responsible for this observation. Since it is known that a weak covalent bond exists between graphene layer and a perfect $\operatorname{Ir}(111)$ single crystal surface, ${ }^{20}$ the presence of grain boundaries might reduce its effect. Therefore, we speculate that the interaction between graphene and $\operatorname{Ir}(111)$ film on sapphire substrate is weaker than that between graphene and $\operatorname{Ir}(111)$ crystal.

The specular rod measurement of the same sample is given in Fig. 9(b), where we clearly observe the satellite fringes of Laue oscillations of the $\operatorname{Ir}(111)$ reflection, similar to that for bare iridium films. A curve is simulated for a single layer graphene on an iridium film on the sapphire substrate, which fits well to the data. Graphene layer is $\sim 0.4 \mathrm{~nm}$ above the iridium top layer, as retrieved from XRR curves and the coherence length for iridium is found to be $14.8 \mathrm{~nm}$. As was observed for as-deposited iridium films, the coherence length is identical to the film thickness derived from the reflectivity curves. Thus, the crystallinity and morphology of the iridium films have been very well preserved after going through the graphene CVD process.

Grazing incidence x-ray diffraction measurements were also conducted on graphene covered iridium films. The in-plane mosaicity was observed to lie in the same range as for as-deposited iridium films. Reciprocal line scans gave similar results compared to that before the synthesis of graphene, and no significant changes in the in-plane and out-ofplane crystallite size and defect structure were observed afterwards.

\section{CONCLUSION}

We have shown that heteroepitaxial $\operatorname{Ir}(111)$ thin films, grown by MBE on (0001) sapphire, act as a suitable support to grow large-area graphene of R0 orientation. We have studied the influence of the growth parameters on the quality of the iridium films deposited on sapphire (0001) single crystals, and subsequently on graphene layers synthesized by CVD on top. Higher iridium deposition rates resulted in the formation of $30^{\circ}$ rotated domains, leading to an incomplete coverage of graphene. Lower growth rates lead to highly crystalline iridium films with atomically smooth surfaces, on which a very homogeneous and a nearly complete coverage of graphene is obtained and the grain boundaries of iridium are limiting the size of the graphene domains. As main defects, the formation of $\mathrm{ABC}$ and $\mathrm{CBA}$ stacked twin domains is observed in the iridium films. 


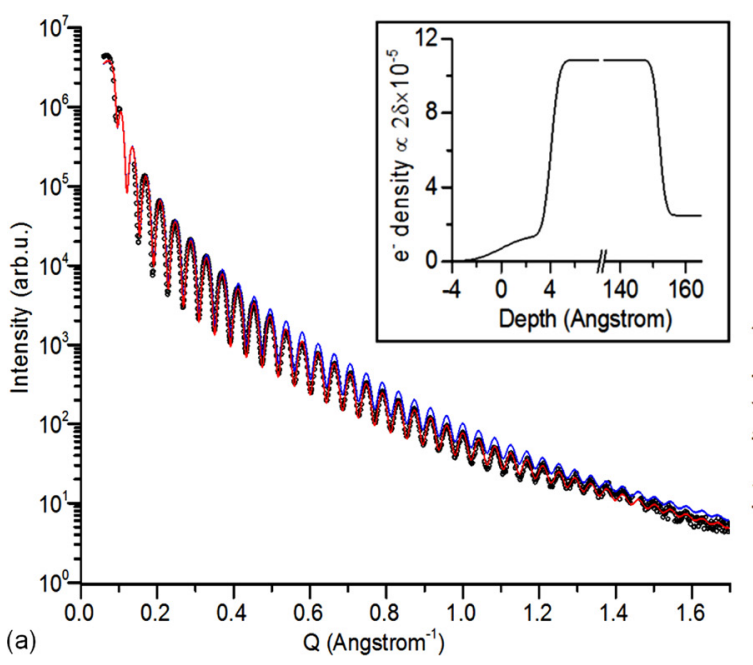

Iridium films were found to be highly stable, and can be re-used for preparing fresh graphene, as done with bulk crystals. Analogous to the graphene moiré on metal single crystals, this system can be utilized for growing metal cluster superlattices on top. This opens the avenue for future studies in which processing of iridium films is involved. In conclusion, our work presented here shows that MBE grown (111) oriented iridium films on sapphire substrates may serve as good substitute for the expensive bulk single crystals presently used for large-area epitaxial graphene growth.

${ }^{1}$ K. S. Novoselov, V. I. Fal'ko, L. Colombo, P. R. Gellert, M. G. Schwab, and K. Kim, Nature (London) 490, 192 (2012).

${ }^{2}$ A. K. Geim, Science 324, 1530 (2009).

${ }^{3}$ X. Li, W. Cai, J. An, S. Kim, J. Nah, D. Yang, R. Piner, A. Velamakanni, I. Jung, E. Tutuc, S. K. Banerjee, L. Colombo, and R. S. Ruoff, Science 324, 1312 (2009).

${ }^{4}$ J. Wintterlin and M. L. Bocquet, Surf. Sci. 603, 1841 (2009).

${ }^{5}$ A. Reina, X. Jia, J. Ho, D. Nezich, H. Son, V. Bulovic, M. S. Dresselhaus, and J. Kong, Nano Lett. 9, 30 (2009).

${ }^{6}$ R. van Gastel, A. T. N'Diaye, D. Wall, J. Coraux, C. Busse, N. M. Buckanie, F. J. Meyer zu Heringdorf, M. Horn von Hoegen, T. Michely, and B. Poelsema, Appl. Phys. Lett. 95, 121901 (2009).

${ }^{7}$ A. T. N'Diaye, J. Coraux, T. Plasa, C. Busse, and T. Michely, New J. Phys. 10, 043033 (2008).

${ }^{8}$ J. Coraux, A. T. N'Diaye, M. Engler, C. Busse, D. Wall, N. Buckanie, F. J. Meyer zu Heringdorf, R. van Gastel, B. Poelsema, and T. Michely, New J. Phys. 11, 023006 (2009).

${ }^{9}$ P. W. Sutter, J. I. Flege, and E. A. Sutter, Nat. Mater. 7, 406 (2008).

${ }^{10}$ E. Loginova, N. C. Bartelt, P. J. Feibelman, and K. F. McCarty, New J. Phys. 10, 093026 (2008).

${ }^{11}$ S. Bae, H. Kim, Y. Lee, X. Xu, J. S. Park, Y. Zheng, J. Balakrishnan, T. Lei, H. R. Kim, Y. I. Song, Y. J. Kim, K. S. Kim, B. Ozyilmaz, J. H. Ahn, B. H. Hong, and S. Iijima, Nat. Nanotechnol. 5, 574 (2010).

${ }^{12}$ A. B. Preobrajenski, M. L. Ng, A. S. Vinogradov, and N. Martensson, Phys. Rev. B 78, 073401 (2008).

${ }^{13}$ I. Pletikosic, M. Kralj, P. Pervan, R. Brako, J. Coraux, A. T. N'Diaye, C. Busse, and T. Michely, Phys. Rev. Lett. 102, 056808 (2009).

${ }^{14}$ P. Sutter, J. T. Sadowski, and E. Sutter, Phys. Rev. B 80, 245411 (2009).

${ }^{15}$ A. Dahal and M. Batzill, Nanoscale 6, 2548 (2014).

${ }^{16}$ L. Meng, R. Wu, L. Zhang, L. Li, S Du, Y. Wang, and H. J. Gao, J. Phys.: Condens. Matter 24, 314214 (2012).

${ }^{17}$ T. A. Land, T. Michely, R. J. Behm, J. C. Hemminger, and G. Comsa, Surf. Sci. 264, 261 (1992).

${ }^{18}$ E. Loginova, S. Nie, K. Thürmer, N. C. Bartelt, and K. F. McCarty, Phys. Rev. B 80, 085430 (2009).

${ }^{19}$ A. T. N'Diaye, S. Bleikamp, P. J. Feibelman, and T. Michely, Phys. Rev. Lett. 97, 215501 (2006).
FIG. 9. (a) X-ray reflectivity curve measured on a graphene covered $15 \mathrm{~nm} \operatorname{Ir}(111)$ film on sapphire (0001). Black circles are the measured data and the fit to the reflectivity curve is plotted in red. For comparison, a simulated curve without considering a graphene layer on the iridium film is plotted in blue. The inset shows the electron density profile from top to bottom of the graphene on iridium film on the sapphire substrate, as derived from the fit. (b) Out-of-plane Bragg scan of $\operatorname{Ir}(111)$ reflection, with the data points shown in black and a simulated curve in red.
${ }^{20}$ C. Busse, P. Lazić, R. Djemour, J. Coraux, T. Gerber, N. Atodiresei, V. Caciuc, R. Brako, A. T. N’Diaye, S. Blügel, J. Zegenhagen, and T. Michely, Phys. Rev. Lett. 107, 036101 (2011).

${ }^{21}$ A. L. Vazquez de Parga, F. Calleja, B. Borca, M. C. G. Passeggi, J. J. Hinarejos, F. Guinea, and R. Miranda, Phys. Rev. Lett. 100, 056807 (2008).

${ }^{22}$ L. Gao, J. R. Guest, and N. P. Guisinger, Nano Lett. 10, 3512 (2010).

${ }^{23}$ A. T. N'Diaye, T. Gerber, C. Bussse, J. Myslivecek, J. Coraux, and T. Michely, New J. Phys. 11, 103045 (2009).

${ }^{24}$ C. Vo-Van, A. Kimouche, A. Reserbat-Plantey, O. Fruchart, P. BayleGuillemaud, N. Bendiab, and J. Coraux, Appl. Phys. Lett. 98, 181903 (2011).

${ }^{25}$ P. Zeller, S. Dänhardt, S. Gsell, M. Schreck, and J. Winterlin, Surf. Sci. 606, 1475 (2012).

${ }^{26}$ C. Struzzi, N. Verbitskiy, A. Fedorov, A. Nefedov, O. Frank, M. Kalbac, G. Di Santo, M. Panighel, A. Goldoni, J. Gärtner, W. Weber, M. Weinl, M. Schreck, Ch. Wöll, H. Sachdev, A. Grüneis, and L. Petaccia, Carbon 81, 167 (2015).

${ }^{27}$ K. Verguts, B. Vermeulen, N. Vrancken, K. Schouteden, C. Van Haesendonck, C. Huyghebaert, M. Heyns, S. De Gendt, and S. Brems, J. Phys. Chem. C 120, 297 (2016).

${ }^{28}$ Z. Fogarassy, M. H. Rümmeli, S. Gorantla, A. Bachmatiuk, G. Dobrik, K. Kamarás, L. P. Biró, K. Havancsák, and J. L. Lábár, Appl. Surf. Sci. 314, 490 (2014).

${ }^{29}$ B. Hu, H. Ago, Y. Ito, K. Kawahara, M. Tsuji, E. Magome, K. Sumitani, N. Mizuta, K. Ikeda, and S. Mizuno, Carbon 50, 57 (2012).

${ }^{30}$ D. L. Miller, M. W. Keller, J. M. Shaw, A. N. Chiaramonti, and R. R. Keller, J. Appl. Phys. 112, 064317 (2012).

${ }^{31}$ R. Vargas, T. Goto, W. Zhang, and T. Hirai, Appl. Phys. Lett. 65, 1094 (1994).

${ }^{32}$ E. Vlieg, J. Appl. Crystallogr. 33, 401 (2000).

${ }^{33}$ L. G. Paratt, Phys. Rev. 95, 359 (1954).

${ }^{34}$ L. Névot and P. Croce, Rev. Phys. Appl. 15, 761-779 (1980).

${ }^{35}$ According to the Scherrer equation, the crystallite size $\left(D_{h k l}\right)$ in the direction perpendicular to the lattice planes of miller indices $h k l$ is given by $D_{h k l}=K \cdot \lambda /\left(B_{h k l} \cdot \cos \theta\right)$, where $\mathrm{K}$ is a numerical factor $(\sim 0.9), \lambda$ is the wavelength of the X-rays, $B_{h k l}$ is the full-width at half-maximum of the $\mathrm{X}$-ray diffraction peak in radians and $\theta$ is the Bragg angle.

${ }^{36}$ R. I. Barabash, W. Donner, and H. Dosch, Appl. Phys. Lett. 78(4), 443 (2001).

${ }^{37}$ R. F. C. Farrow, G. R. Harp, R. F. Marks, T. A. Rabedau, M. F. Toney, D. Weller, and S. S. P. Parkin, J. Cryst. Growth 133, 47 (1993).

${ }^{38}$ H. Zhou, P. Wochner, A. Schöps, and T. Wagner, J. Cryst. Growth 234, 561 (2002).

${ }^{39}$ S. Bleikamp, J. Coraux, O. Robach, G. Renaud, and T. Michely, Phys. Rev. B 83, 064103 (2011).

${ }^{40}$ F. Jean, T. Zhou, N. Blanc, R. Felici, J. Coraux, and G. Renaud, Phys. Rev. B 88, 165406 (2013).

${ }^{41}$ H. Hattab, A. T. N'Diaye, D. Wall, G. Jnawali, J. Coraux, C. Busse, R. van Gastel, B. Poelsema, T. Michely, F. J. Meyer zu Heringdorf, and M. Horn-von Hoegen, Appl. Phys. Lett. 98, 141903 (2011).

${ }^{42}$ D. Franz, S. Runte, C. Busse, S. Schumacher, T. Gerber, T. Michely, M. Mantilla, V. Kilic, J. Zegenhagen, and A. Stierle, Phys. Rev. Lett. 110, 065503 (2013). 